\title{
Handlungsansätze und Strategien im Umgang mit Multilokalität
}

\section{Lena Greinke, Franziska Lehmann, Frank Othengrafen und Annette Seitz}

Multilokalität ist eine Lebensweise, die nahezu in allen Altersgruppen und Gesellschaftsschichten vertreten ist (vgl. Beitrag Greinke/Lange/Born in diesem Band (Kap. 2)). Allerdings fehlen den lokalen Akteur*innen oftmals das Wissen und Bewusstsein über die Zusammenhänge und Auswirkungen multilokaler Lebensweisen, Multilokalität wird häufig wenig bis gar nicht wahrgenommen. Somit haben die Akteur*innen zumeist (noch) keine Handlungsbedarfe identifiziert oder Handlungsoptionen entwickelt. Um aber auf das zunehmende Phänomen Multilokalität reagieren zu können sowie geeignete Strategien und Ansätze zu entwickeln, ist es bedeutsam, die relevanten Akteur*innen für Multilokalität und die daraus resultierenden Auswirkungen dieser Lebensweisen zu sensibilisieren (siehe Abb. 7.1).

\footnotetext{
L. Greinke $(\bowtie)$

Institut für Umweltplanung, Leibniz Universität Hannover, Hannover, Deutschland E-Mail: greinke@umwelt.uni-hannover.de

F. Lehmann proloco - Bremen, Bremen, Deutschland

E-Mail: lehmann@proloco-bremen.de

F. Othengrafen

Fachgebiet Stadt- und Regionalplanung, Technische Universität Dortmund, Dortmund, Deutschland

E-Mail: frank.othengrafen@tu-dortmund.de
}
A. Seitz
Landkreis Diepholz, Diepholz, Deutschland
E-Mail: annette.seitz@diepholz.de

(C) Der/die Autor(en) 2021

F. Othengrafen et al. (Hrsg.), Temporäre An- und Abwesenheiten in ländlichen

Räumen, https://doi.org/10.1007/978-3-658-32500-8_7 


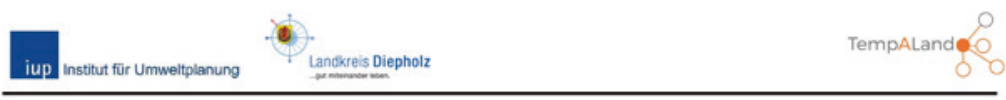

\section{The News}

Kreisblatt TempAHausen

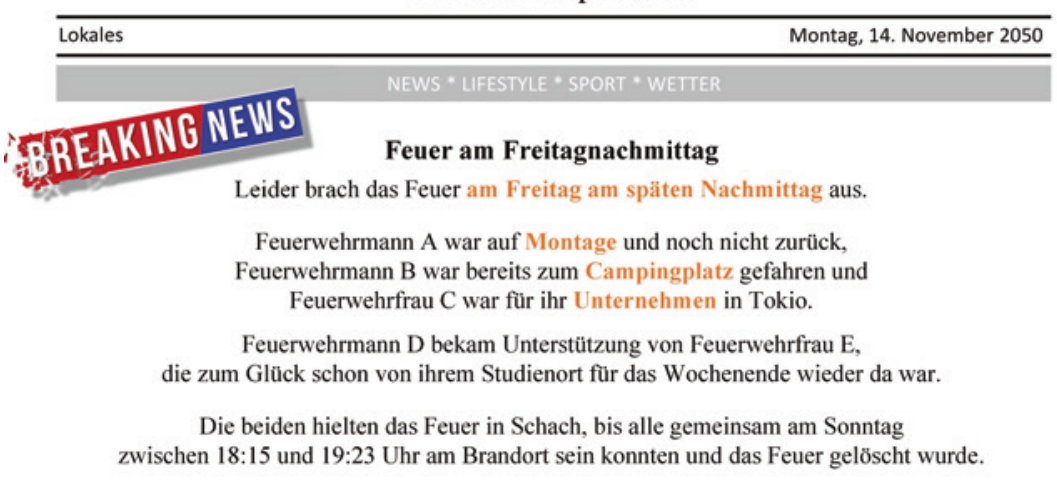

Abb.7.1 Fiktive Schlagzeile aus dem Kreisblatt TempAHausen im Jahr 2050 (Quelle: TempALand 2020)

In diesem Kontext wurden im Projekt TempALand verschiedene Instrumente und Formate entwickelt, erprobt und auf ihre Übertragbarkeit getestet, um (1) für Multilokalität zu sensibilisieren, (2) die Wahrnehmung des Phänomens zu erhöhen und (3) geeignete Strategien und Ansätze im Umgang mit Multilokalität zu entwickeln. Dabei haben sich besonders diaologorientierte und spielerische Zugänge zur Bewusstseinsbildung als geeignet herausgestellt, um Akteur*innen aus Gesellschaft, Planung, Politik, Vereinen, Institutionen und Wirtschaft etc. unterschiedliche Einblicke und Perspektiven zu ermöglichen. Nachfolgend werden zunächst die im Projekt TempALand eingesetzten Instrumente und Formate - Gesellschaftsspiel (Kap. 7.1.1), Beteiligungsformate (Kap. 7.1.2), Veranstaltungsreihe (Kap. 7.1.3) und Planspiel (Kap. 7.1.4) - vorgestellt und deren Chancen und Risiken erläutert, bevor beispielhaft ausgewählte Handlungsansätze und Strategien zum Umgang mit Multilokalität in der Praxis (Kap. 7.2 und 7.3) vorgestellt werden. 


\subsection{Annäherung an eine „Unbekannte“ - Sensibilisierung für Multilokalität}

Die verschiedenen spielerischen und partizipativen Formate verfolgen unterschiedliche Ziele: Bei einigen Ansätzen stehen eher Information und Bewusstseinsbildung für das Thema „Multilokalität“ im Vordergrund, bei anderen Formaten geht es um die Identifizierung der mit multilokalen Lebensweisen verbundenen (lokalen und regionalen) Herausforderungen und bei wiederum anderen Ansätzen wird die gemeinsame Entwicklung von Lösungsvorschlägen verfolgt. Die verschiedenen Ansätze und Formate ergänzen sich und können, je nach Ausgangslage und Akteurskonstellation, unterschiedlich miteinander kombiniert werden. In der Gesamtschau dienen die einzelnen Ansätze und Formate aber allesamt dazu, Akteur*innen aus Gesellschaft, Planung, Politik, Vereinen, Institutionen und Wirtschaft etc. unterschiedliche Einblicke und Perspektiven zu ermöglichen und Handlungsansätze zu entwickeln.

\subsubsection{Worum geht es bei Multilokalität? - Ein Gesellschaftsspiel als Einstieg in das Thema}

Multi-was? Was bedeutet Multilokalität? Wer sind Multilokale? Was sind Motive und Gründe für ein Leben an mehreren Orten? Das Gesellschaftsspiel „Multilokalität" (siehe Abb. 7.2) sensibilisiert spielerisch und niedrigschwellig für die Alltagswelt multilokal Lebender und steht unter www.tempaland.de kostenlos zum Download zur Verfügung. Es eignet sich v. a. bei öffentlichen Veranstaltungen, indem es zu Beginn während der Registrierung, der Begrüßung oder in Pausen von Veranstaltungen ausgelegt wird und die Teilnehmer*innen über das Gesellschaftsspiel gemeinsam mehr über die Herausforderungen und Potenziale des Lebens an mehreren Orten erfahren. Somit kann das Spiel das Bewusstsein für Multilokalität bei den Teilnehmer*innen erhöhen und zu einer Sensibilisierung beitragen. 


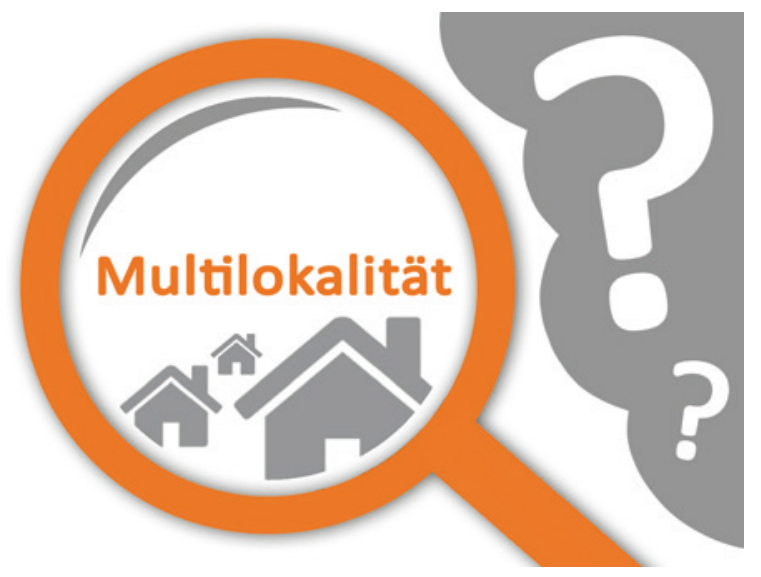

Abb. 7.2 „Gesellschaftsspiel“: Worum geht es bei Multilokalität? (Quelle: TempALand 2020)

\section{Gesellschaftsspiel „Multilokalität"}

Es ist als Leiterspiel mit zwei bis vier Personen innerhalb von ca. 20 bis $30 \mathrm{~min}$ zu spielen. Neben dem Spielplan (siehe Abbildung) besteht das Spiel aus Ereigniskarten, Spielfiguren und einem Augenwürfel. Die Spieler*innen erhalten jeweils eine Spielfigur und platzieren diese auf dem Startfeld. Der jüngste Spieler oder die jüngste Spielerin beginnt, danach wird im Uhrzeigersinn gewürfelt und die Augenzahl vorgerückt. Landet die Spielfigur am Ende des Zuges auf einer Leiter oder Rutsche, rückt die Figur auf der Leiter vor oder auf der Rutsche zurück. Landet die Spielfigur auf einem Aktionsfeld, muss eine Ereigniskarte gezogen werden. Gewonnen hat die Spielfigur, die als erstes das Zielfeld erreicht. Für den Zieleinzug ist jedoch die passende Augenzahl nötig, überzählige Punkte müssen ansonsten rückwärtsgegangen werden. 


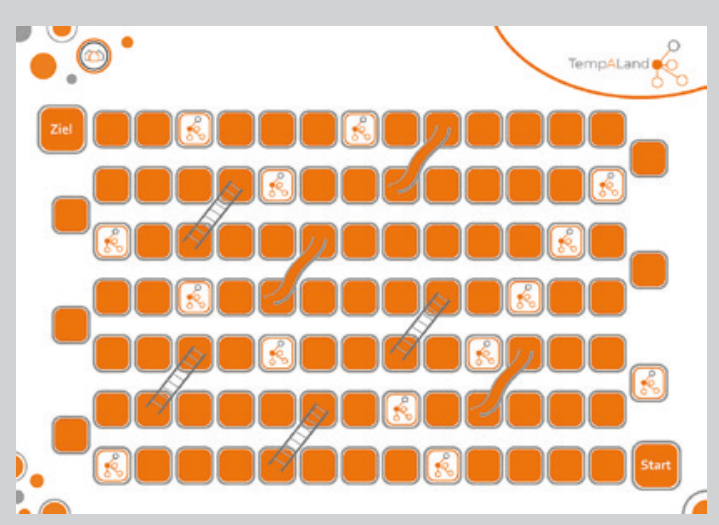

Die Ereigniskarten stellen beispielhaft Situationen und Ereignisse aus dem Lebensalltag von Multilokalen dar. Dabei werden unter anderem Themen aus den Handlungsfeldern Unternehmen, soziale und technische Infrastrukturen, bürgerschaftliches Engagement, Wohnen und Kommunen in den Karten aufgegriffen:

Stellen Sie sich vor, Sie reisen zwischen Ihren Orten mit dem Auto hin und her (siehe Abbildung). Bei einer Inspektion Ihres Autos wird ein Defekt festgestellt. Damit Sie nun pünktlich zur Arbeit an Ihren weit entfernten Arbeitsplatz gelangen, müssen Sie einen Mietwagen nehmen. Das kostet Sie viel Zeit, weil Sie zunächst einen Mietwagen organisieren und sich parallel um die Reparatur Ihres Autos kümmern müssen. Durch die Anmietung eines zusätzlichen Wagens entstehen höhere Kosten für Sie. Diese Situation ist für multilokal Lebende nicht zwingend alltäglich, kommt aber vor und stellt die Personen vor besondere Herausforderungen in ihrer Alltagsorganisation. 


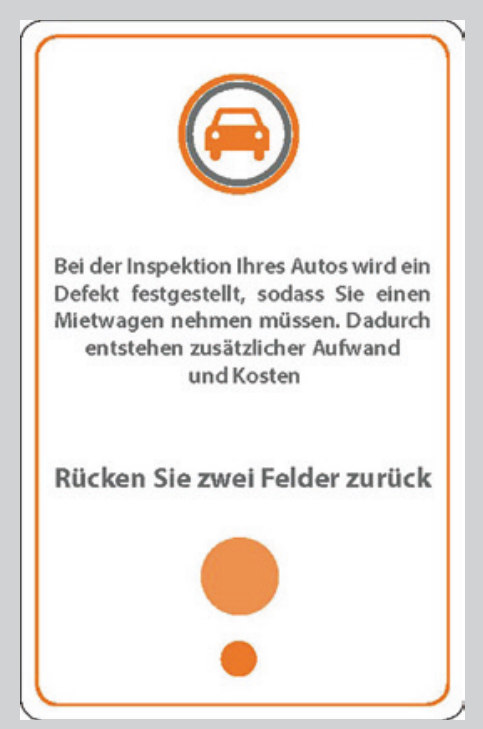

Ihr Arbeitgeber und die Kommunen unterstützen Multilokale bei der Wohnungs- oder Arbeitsplatzsuche. Dadurch konnte Ihre Partnerin oder Ihr Partner eine Anstellung in der Nähe Ihres Arbeitsplatzes finden. Nun können Sie beide umziehen und somit mehr Zeit miteinander verbringen. Durch dieses neue Arrangement geben Sie ihre multilokale Lebensweise auf und verbringen mehr Zeit mit Ihrer Familie, Ihrem Partner oder Ihrer Partnerin und finden neue Freund*innen. 


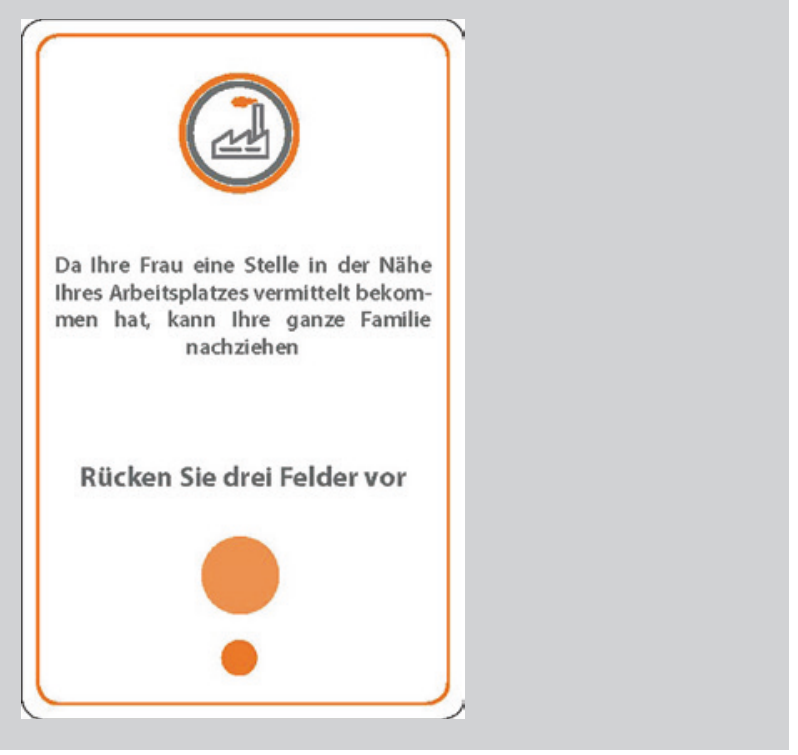

An Grenzen stößt das Spiel, wenn es darum geht, das Phänomen tiefgreifend $\mathrm{zu}$ verstehen und Auswirkungen detailliert nachzuvollziehen. Als Einstieg in die Thematik ist es für nahezu alle Altersgruppen und Gesellschaftsschichten geeignet; es soll Interesse wecken für weitere Anschlussformate und instrumente, damit für Multilokalität ausreichend sensibilisiert wird.

\subsubsection{Zusammenarbeit mit einzelnen Zielgruppen: Akteursgespräche und Workshops}

Der Umgang mit Multilokalität legt das Zusammenwirken verschiedener Akteursgruppen nahe, damit besitzen zielgruppenspezifische Gesprächs- und Mitwirkungsangebote eine große Bedeutung. Das Thema Multilokalität ist ein relativ neues Thema und akuter Handlungsdruck wird - im Vergleich zu anderen (kommunalpolitischen) Themen wie dem demografischen Wandel, dem Fachkräftemangel, der Digitalisierung oder dem Klimawandel - nicht als vorrangiges Thema gesehen. Von daher ist die Resonanz auf die Einladung zu öffentlichen Veranstaltungen i. d. R. eher begrenzt, sodass zielgruppenspezifische Ansprachen erforderlich sind. Im Projekt TempALand konnten für eine öffentliche Auftaktveranstaltung zur Vorstellung und Diskussion des Projektes TempALand im 
Landkreis Diepholz etwa 80 Interessierte gewonnen werden, mit denen erste Diskussionen zu Multilokalität im Landkreis Diepholz geführt wurden. Im weiteren Projektablauf wurde dann auf zielgruppenspezifische Mitwirkungsund Beteiligungsangebote gesetzt. Für die Teilnahme an diesen Veranstaltungen wurde häufig mit persönlicher Ansprache einzelner Personen ,geworben“. Dabei hat sich das Zusammenwirken lokaler Akteur*innen (aus Verwaltung und Kommunalpolitik) mit dem wissenschaftlichen Projektteam bewährt. Das sowohl aus der Praxis wie aus der Wissenschaft geäußerte Interesse am Thema konnte lokale Akteur*innen von einer Mitwirkung überzeugen. Dabei mussten die Erwartungshaltungen an das Engagement der lokalen Akteur*innen dem bestehenden - oder nicht bestehenden - Handlungsdruck durch Multilokalität im ländlichen Raum angepasst werden.

Im ersten Schritt wurden Akteursgespräche mit jeweils einem kleineren Kreis aus einer Akteursgruppe (Wohnungswirtschaft, Unternehmen, Vereine und Verbände) geführt. Die Gespräche dienten der Kontaktaufnahme und der Sensibilisierung für das Thema. Darauf aufbauend wurden im Rahmen von Workshops mit Vertreter*innen verschiedener Akteursgruppen Handlungsansätze zu den Themen Wohnen, Unternehmen und Engagement entwickelt (siehe Abb. 7.3). Diese wurden anschließend vom Projektteam zu Projektskizzen verdichtet und im Rahmen eines gemeinsamen Workshops mit ca. 40 Teilnehmer*innen diskutiert, weiterentwickelt und priorisiert.

"Zielgruppenspezifische Angebote" umfassten im TempALand-Prozess sowohl das Arbeiten mit einzelnen Zielgruppen als auch das gezielte Zusammenbringen und den Austausch zwischen verschiedenen Akteursgruppen, um die gegenseitigen Handlungsspielräume, Themenschwerpunkte und Rahmensetzungen kennen und verstehen zu lernen. So erarbeiteten z. B. im Workshop zum Thema Wohnen Vertreter*innen der Wohnungswirtschaft in einer Arbeitsphase Handlungsansätze für die öffentliche Hand und umgekehrt Vertreter*innen

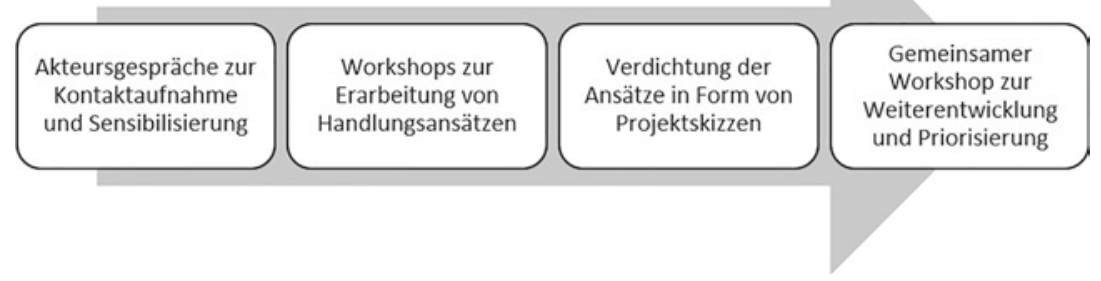

Abb. 7.3 Dialogprozess im Projekt TempALand (Quelle: TempALand 2020) 


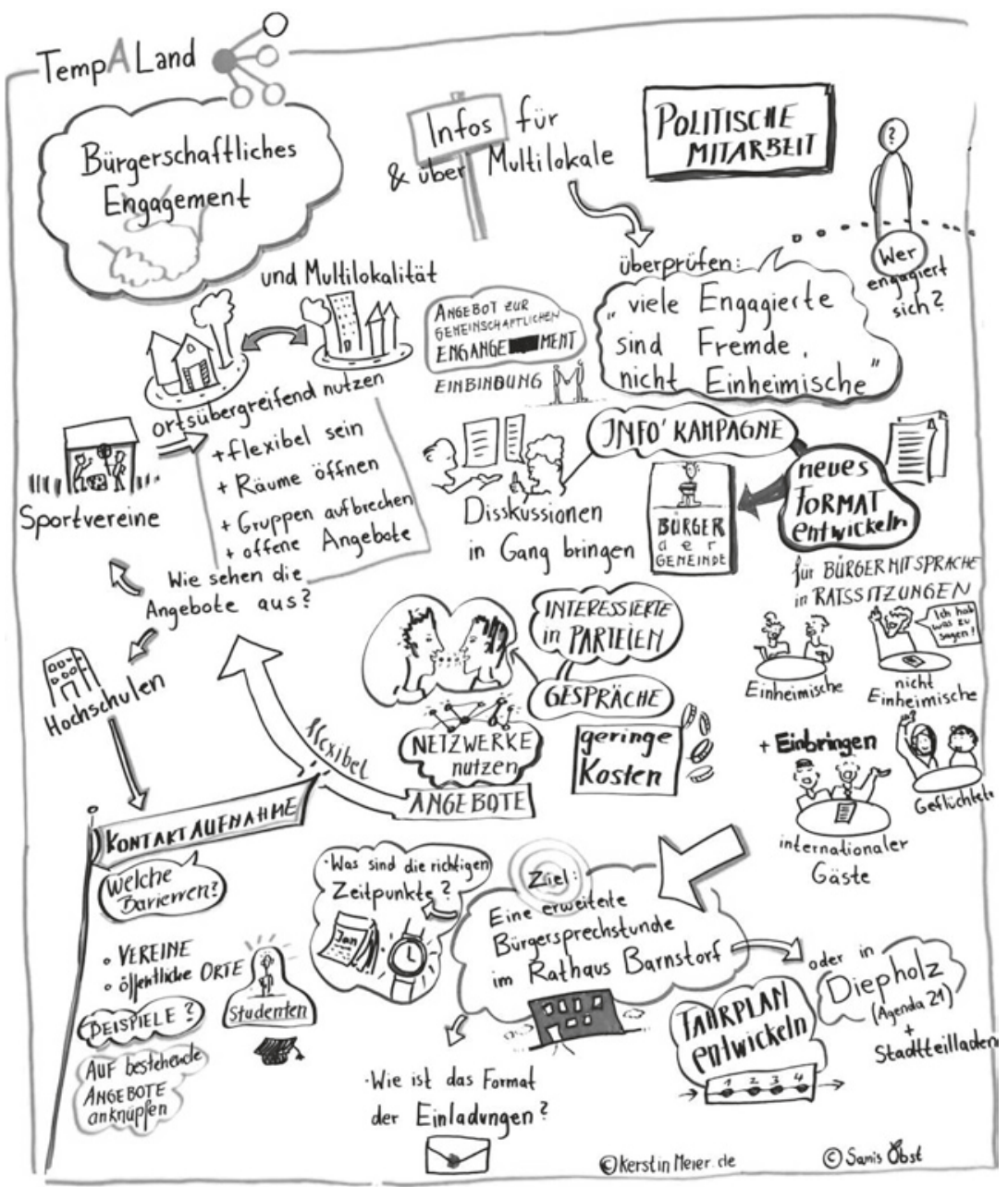

Abb. 7.4 Handlungsansätze im Themenfeld „Bürgerschaftliches Engagement und Multilokalität“ - Ergebnisse eines gemeinsamen Workshops verschiedener Zielgruppen (Quelle: Kerstin Meyer \& Samis Obst, TempALand 2020)

der öffentlichen Hand Handlungsansätze für die Wohnungswirtschaft. Der anschließende Austausch und die Diskussion mit den ,echten“ Vertreter*innen der jeweiligen Akteursgruppe erbrachten zum einen Transparenz und wechselseitiges Verständnis, zum anderen qualitativ gute und tragfähige Ergebnisse (Abb. 7.4). 
Die zielgruppenspezifischen Arbeitsformen haben sich im Kontext mit Multilokalität bewährt. Sie ermöglichten es, frühzeitig in kleinen thematischen Gruppen wissenschaftliche Erkenntnisse und fachliches Praxiswissen zu verknüpfen und gerade dadurch praxistaugliche Handlungsansätze zu entwickeln. Die Tatsache, dass der durch Multilokalität verursachte Handlungsdruck für die verschiedenen Akteur*innen (noch) gering ist, hat ggf. mit dazu beigetragen, dass die Entwicklung von Handlungsansätzen kreativ und konstruktiv möglich war, da niemand „direkte“ Konsequenzen für sich bzw. die durch ihn/sie vertretene Institution befürchten musste. Gleichzeitig führten die Diskussionen rund um das Thema Multilokalität und die damit erreichte Sensibilisierung jedoch dazu, dass die beteiligten Akteur*innen bei zukünftig anstehenden Konzepten, Projekten und Entscheidungen das Thema Multilokalität mitdenken und berücksichtigen wollen bzw. werden. Inwieweit das zur Umsetzung konzeptioneller Ansätze und Ideen führen wird, wie sie in Kap. 7.2 beschrieben werden, hängt zum Teil davon ab, inwieweit die Ansätze den alltäglichen Handlungsfeldern der Akteur*innen entsprechen und wie groß der Handlungsdruck im jeweiligen Thema auch unabhängig von der Multilokalität ist. So ist z. B. die Frage der Anpassung des Wohnungsbestandes an aktuelle Nachfragen gerade in ländlichen Räumen ein aktuelles Thema, bei dem es wichtig ist, dass die sensibilisierten Akteur*innen das Thema Multilokalität und die damit verbundenen Auswirkungen auf die Wohnungsnachfrage (durch Incomings) frühzeitig mitdenken.

\subsubsection{Planerische Akteur*innen im Fokus - Wohnraumversorgungskonzept und Zukunftsdialog Wohnungsmarktentwicklung}

Das Thema Wohnen an verschiedenen Orten ist für alle Multilokalen von essenzieller Bedeutung. Am zweiten Wohnort wird in der Regel eine kleinere, möglichst bezahlbare Wohnung oder Unterkunft nachgefragt. Damit verstärken Multilokale die Nachfrage Alleinstehender aller Altersklassen nach Wohnraum, dazu zählen auch Alleinerziehende, Ältere, Geringverdienende, Auszubildende und Studierende (s.a. Beiträge Greinke/Lange (Kap. 4) und Greinke/Albrecht/ Othengrafen/Gutsche/Lehmann (Kap. 5) in diesem Band).

Als Annäherung an und Reaktion auf das Phänomen Multilokalität eignet sich die Erstellung von Konzepten, die explizit mehrörtige Lebensweisen berücksichtigen. Bisher taucht allerdings die so schwierig zu quantifizierende Gruppe der Multilokalen in demografischen oder wohnungsmarktrelevanten Untersuchungen von ländlichen Kommunen und Landkreisen als zu berücksichtigendes 

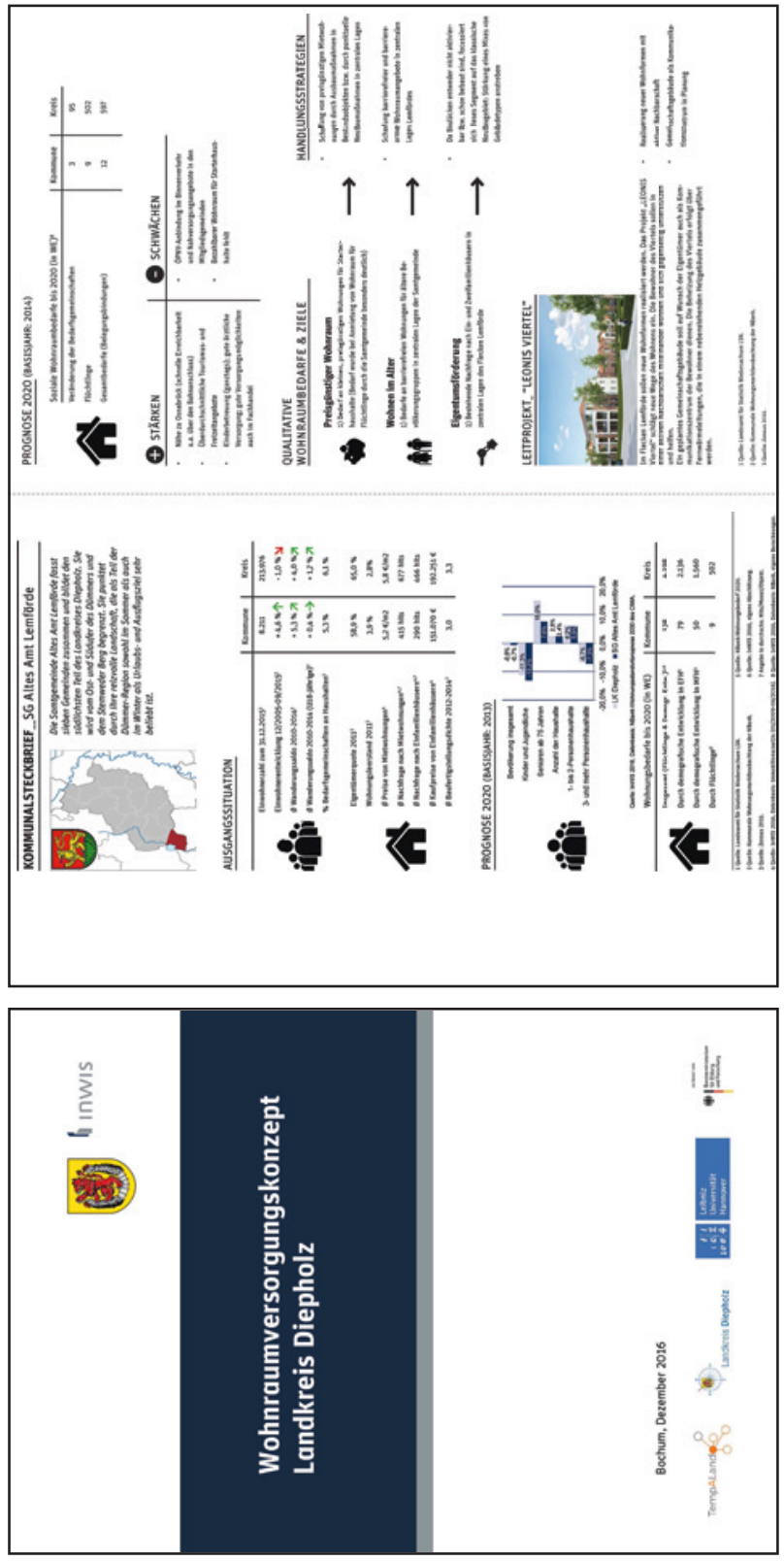

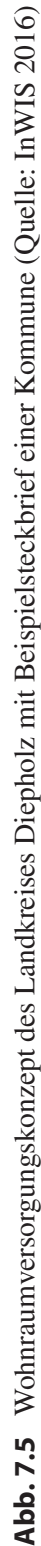


Einwohner*innen-Segment nicht auf. Anders in dem im Landkreis Diepholz im Rahmen des Projektes TempALand entwickelten Wohnraumversorgungskonzept (WRVK), mit dessen Hilfe sowohl der aktuelle Wohnungsmarkt als auch die zukünftigen Entwicklungen im Landkreis Diepholz analysiert wurden (siehe Abb. 7.5). In direkter Abstimmung mit den 15 Kommunen und dem Landkreis wurden dazu u. a. individuelle kommunale Steckbriefe erarbeitet. Zum einen gaben diese den Kommunen einen fundierten Überblick über den aktuellen Wohnungsmarkt und dessen zukünftige Entwicklung zum anderen eine konkrete Planungshilfe an die Hand. Mit Blick auf Multilokale, inbesondere Incomings, konstatiert das WRVK, dass kleine und auch bezahlbare Mietwohnungen im von Ein- und Zweifamilienhäusern geprägten Landkreis weitgehend fehlen. Das ist ein wichtiges Ergebnis für die Entscheidungsträger*innen, nicht zuletzt vor dem Hintergrund, dass der Bedarf nach kleineren und bezahlbaren Mietwohnungen aufgrund der zunehmenden Zahl an Einpersonen-Haushalte weiter zunehmen wird.

Diese und andere Konzepte oder Studien ermöglichen es, die Anforderungen und spezifischen Bedürfnisse multilokal lebender Personen in einen größeren Kontext einzubetten und gezielt an Handlungsansätzen und -strategien in verschiedenen Bereichen (z. B. im Bereich „Wohnen“) zu arbeiten. Darüber hinaus können lokale und regionale Akteur*innen, insbesondere aus den Bereichen Politik und Verwaltung, die im WRVK generierten Erkenntnisse für die Arbeit und Planung vor Ort heranziehen und auf verschiedenen Ebenen nutzen.

Die Erkenntnisse und Prognosen aus dem WRVK des Landkreises Diepholz führten im Projektprozess dazu, dass eine Veranstaltungsreihe konzipiert wurde, um möglichst breit mit auf dem Wohnungsmarkt und in der Planung aktiven Personen über Themen wie Innenentwicklung, Umgang mit Leerständen und Quartieren der 1950er bis 1970er Jahre zu diskutieren. Über diese Themen ließen sich nicht nur die Wohnungsbedarfe der wachsenden Gruppe multilokal Lebender vermitteln, sondern es konnte auch grundsätzlich für das Phänomen von „Teilzeit"-Einwohner*innen sensibilisiert werden. Im Mittelpunkt des Dialogformats wurden gute Beispiele aus externen Kommunen vorgestellt und diskutiert. Für die Planungs- und Bauämter der Kommunen des Landkreises wurden darüber hinaus zwei Planer*innen-Seminare durchgeführt, in denen bspw. anhand der Novellierungen des BauGB und der BauNVO aufgezeigt wurde, wie landwirtschaftliche Gebäude für Wohnzwecke umgenutzt werden können oder wie das sogenannte urbane Wohngebiet ermöglicht wird. 


\subsubsection{Zukünftige Entwicklungen, Herausforderungen und Handlungsansätze erlebbar machen - Planspiel „Zukunftsdialog Multilokalität"}

Die Methode des Planspiels (siehe Abb. 7.6) setzt auf einen kreativ-spielerischen Rahmen, in dem gemeinsam innovative Lösungsansätze entwickelt werden können. „Spielerisch“ meint hier nicht nur einen lockeren, unbefangenen Ansatz, sondern auch eine optimierte Form des Lernens. Die Methode eignet sich dadurch u. a. für Fragestellungen, für die noch keine „Standardlösungen“ vorliegen und Kreativität im Umgang mit dem Thema gefragt ist. Das im Projekt TempALand entwickelte Planspiel „Zukunftsdialog Multilokalität" wurde so konzipiert und aufbereitet, dass es von anderen ,nachgespielt“ werden kann. Auf der Internetseite des Projektes www.tempaland.de liegen die vollständigen Spielmaterialien und eine Spielanleitung zum Download bereit.

Das Planspiel „Zukunftsdialog Multilokalität“ wurde zunächst im Landkreis Diepholz und anschließend zum Vergleich bzw. zur Überprüfung im Landkreis Saalekreis (Sachsen-Anhalt) durchgeführt. Die jeweils etwa 15 Teilnehmer*innen kamen aus Verwaltung und Politik, Wohnungswirtschaft, Vereinen, Feuerwehr etc. Durch den fiktiven, jedoch realitätsnahen Rahmen und das Spielen unterschiedlicher Rollen ergeben sich für die Teilnehmer*innen eines Planspiels folgende Vorteile:

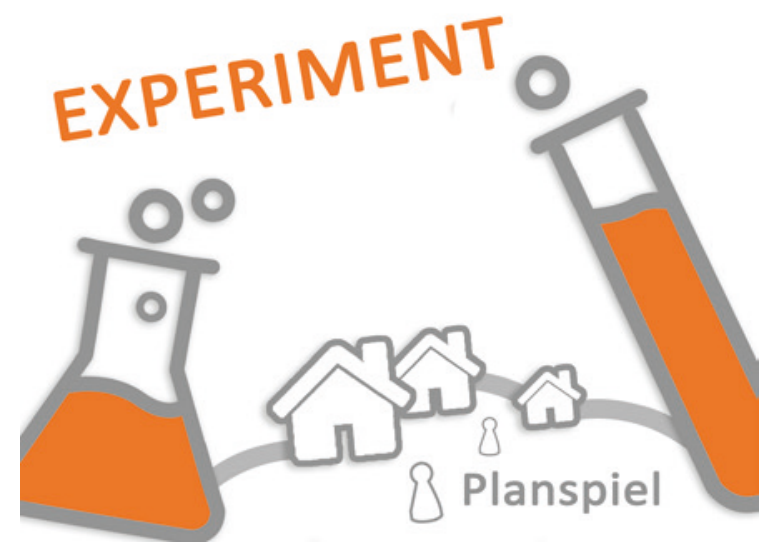

Abb. 7.6 Experiment: Erfahren Sie im Planspiel, was Multilokalität bedeutet (Quelle: TempALand 2020) 
- Änderung des Blickwinkels: Die Übernahme einer Rolle ermöglicht es, auf eine gegebene Fragestellung aus einer neuen Perspektive zu blicken. Zum einen besteht die Chance, Verständnis für andere zu gewinnen. Zum anderen können sich durch neue Sichtweisen auch neue Lösungswege und Lösungen eröffnen.

- Überwinden alltäglicher Routinen: Die Spielsituation hilft, die Ebene dessen, was die Beteiligten ,immer denken“, und ,immer tun“ zu verlassen und offen zu sein für die Aufgabenstellung des Spiels.

- Erhöhung der Fehlertoleranz: Die Spielsituation und die Übernahme einer Rolle geben gerade Fachleuten die Freiheit, auch Dinge zu sagen und auszuprobieren, bei denen nicht sicher ist, ob sie stichhaltig und fehlerfrei sind. Eine Unternehmerin aus dem fiktiven Kreis Bruchfurt kann im Planspiel Dinge frei äußern und Konzepte ausprobieren, die eine Frau aus der realen Kommunalverwaltung nie sagen oder tun und ggf. nicht einmal denken würde. Dadurch können sich neue und kreative Gedankenwege und Lösungen ergeben.

- Freude, Spannung und Vergnügen als Voraussetzung für innovatives und kreatives Denken: Bei entsprechender Vorbereitung und Spielfreude der Teilnehmenden führt die Spielsituation dazu, dass auch ein Thema ohne akuten Handlungsdruck plötzlich mit Engagement, Freude, Spannung und Vergnügen durchdacht und bearbeitet wird. Alle Beteiligten wissen, dass das Planspiel eine temporäre Sondersituation ist, doch der Ernst der Sache wird keineswegs aus den Augen verloren.

\section{Das Planspiel „Zukunftsdialog Multilokalität“}

Grundlage für das Planspiel ist der fiktive Landkreis Bruchfurt. Im Jahr 2025 leben dort $20 \%$ der Menschen multilokal. Aktuelle Abschätzungen der Zahl der multi-lokal Lebenden Menschen z. B. im Landkreis Diepholz liegen unter $10 \%$. Im Planspiel wird dieser Anteil bewusst höher gesetzt, um das Thema Multilokalität und seine Folgen besser sichtbar machen zu können. 


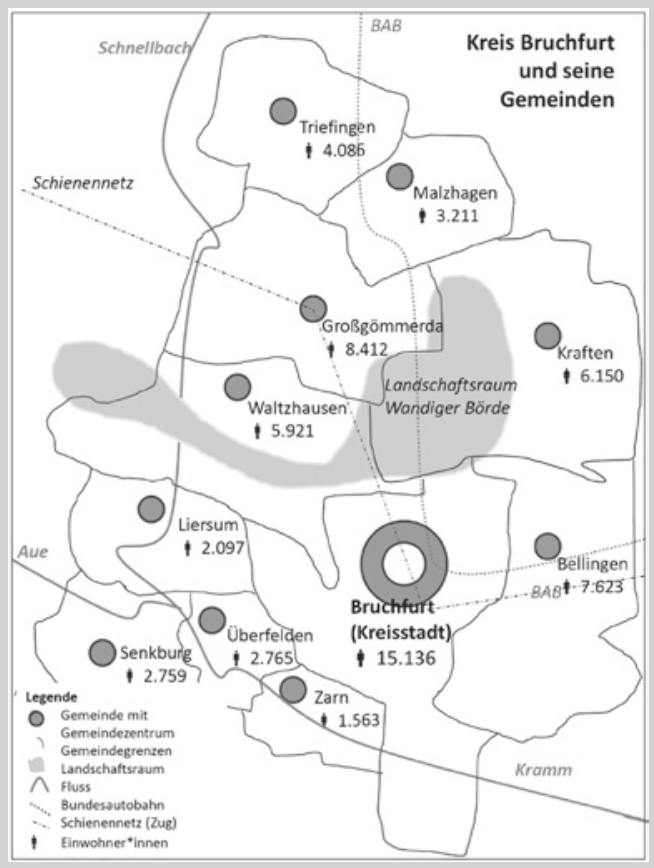

Die Spielenden nehmen Rollen aus den drei Interessensgruppen „Verwaltung und Politik“, „Unternehmen“ und „Vereine, Verbände und Initiativen“ ein. Rollenprofile mit biographischen Angaben und dem jeweiligen Bezug zur Multilokalität ermöglichen einen Rollenwechsel und damit einen Wechsel des Blickwinkels. Das Spielmaterial umfasst quantitative und qualitative Angaben, die den Landkreis Bruchfurt und die Multilokalität in relativ knapper, anschaulicher Form beschreiben (s.a. Beschreibung des Planspiels unter www.tempaland.de). Das Spielmaterial ist möglichst realitätsnah und bildhaft gestaltet, um den Teilnehmenden einen schnellen Einstieg in die Fiktion des Spiels zu ermöglichen. Die Entscheidung für eine fiktive, realitätsnahe Fallkonstellation wurde bewusst getroffen, um die Rahmenbedingungen des Spiels auf die Zielsetzungen auszurichten und eine im Verlauf des Spiels lösbare Aufgabe definieren zu können.

Die Spieldauer ist auf vier Stunden begrenzt. In dieser Zeit erarbeiten die drei Interessensgruppen erst getrennt Handlungsvorschläge und einigen sich anschließend in einer gemeinsamen Sitzung auf die Maßnahmen, die in Bruchfurt in nächster Zeit umgesetzt werden sollen. So werden der 
Nutzen und die Möglichkeiten abgestimmter und gemeinsamer Handlungsansätze im Umgang mit Multilokalität deutlich.

\section{Alexander Rosche}

Alexander Rosche, 31, ist Versicherungsvertreter und im Vereinsvorstand des FC Bruchfurt. Er ist leidenschaftlicher Fußballer und trainiert die BJunioren im Verein. Auch als Schiri findet man ihn hin und wieder auf dem Platz.

Er ist alteingesessener Bruchfurter und lebt mit seiner Freundin zentral am alten Markt. Durch seine Arbeit, den Verein und seine Familie ist er sehr gut vernetzt. Der Attraktivitätsverlust Bruchfurts bereitet inm Sorgen.

FC Bruchfurt musste 2035 seine bis dahin sehr erfolgreiche Teilnahme an der Kreisliga wegen akutem Spielermangel aufgeben. Gespräche mit dem Verband über eine Änderung der Bedingungen (Spieleranzahl) und mit dem TUS Spielholz 1890 (Nachbarkreis) über eine Zusammenlegung werden zur Zeit geführt.

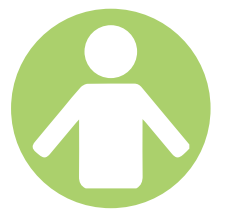

FC Bruchfurt e.V. Vereinsvorstand

Multilokalität

- Auswirkung auf Vereinsleben

- weniger Nachwuchs

- Trainingsausfall

- Vereinsaufgaben können nicht mehr wahrgenommen werden

Das Planspiel hat sich im Kontext des Themas Multilokalität bewährt. Die Methode wurde sowohl im Landkreis Diepholz als auch im Saalekreis von den Teilnehmer*innen engagiert, mit viel Spielfreude und ergebnisorientiert angenommen. So äußerte eine Teilnehmerin an dem Planspiel im Landkreis Diepholz in der Feedback-Runde: „Ich habe ja in den letzten zwei Jahren immer mal wieder von dem Projekt gehört, aber jetzt habe ich verstanden, worum es dabei geht und warum es ein Thema für uns ist." Ein anderer Teilnehmer fasste zusammen, dass das Planspiel für ihn die „einzig sinnvolle Methode“ sei. Der konstruktive, kreative und entspannte Ablauf der beiden Planspiele mit inhaltlich tragfähigen Ergebnissen bestätigt die auch in anderen Projekten gesammelten positiven Erfahrungen mit der Methode Planspiel im Kontext planerischer Fragestellungen und Themenstellungen, bei denen es (noch) keine "Standardlösungen“ gibt. Im Rahmen vom Projekt TempALand besonders hervorzuheben ist die Tatsache, dass die Vertreter*innen des Saalekreises nach dem Planspiel geäußert haben, dass sie das Thema Multilokalität in verschiedenen Zusammenhängen (Wohnraumversorgungskonzept, Mobilitätskonzept usw.) stärker berücksichtigen wollen. Der reibungslose Ablauf der beiden Planspiele legt zudem nahe, dass das Planspiel von anderen Akteur*innen mithilfe des zur Verfügung gestellten Spielmaterials umgesetzt werden kann (www.tempaland.de) (Abb. 7.7). 


\section{HILLSTADT - BOTE}

\section{e-news since 2019}

IN DER DISKUSSION

04.04.2025

\section{Bruchfurt diskutiert, heute: Unfreiwillige Feuerwehr}

An dieser Stelle wie jede Woche pro- und kontra-Stimmen zu einer aktuellen Diskussion:

Seit kurzer Zeit wird im Landkreis Bruchfurt - und nicht nur hier, auch in anderen Landkreisen der Region - über die Frage diskutiert, ob dem chronischen Personalmangel der freiwilligen Feuerwehr durch Einführung einer unfreiwilligen Feuerwehr begegnet werden kann. Öffentliche Verwaltungen und Unternehmen mit mehr als 50 Beschäftigten sollen pro 50 Arbeitnehmer*innen eine*n Mitarbeiter*in für die Arbeit in der Feuerwehr qualifizieren und bei Bedarf freistellen.

Pro:

Markus Möllering, Kreistagsabgeordneter

„Die Nachwuchssorgen der freiwilligen Feuerwehren im Kreis sind inzwischen nahezu sprichwörtlich. Dass es dadurch noch zu keinen ernsthaften Schäden an Leben und Besitz gekommen ist, muss als ein Wunder gelten.

Es ist an der Zeit, sich davon zu verabschieden, dass Freiwilligkeit alleine ausreicht, diese wichtige gesellschaftliche Aufgabe wahrzunehmen. Wenn Leben in Gefahr sind, können wir uns das nicht mehr leisten. Arbeitgeber*innen und ihre Beschäftigten hier mit in die Verantwortung zu nehmen, ist der richtige Weg und auch in ihrem eigenen Interesse.

Unsere Fraktion hat diesen Vorschlag nicht zuletzt deshalb eingebracht, um der ermüdend gleichbleibenden Diskussion um die Freiwilligen Feuerwehren endlich einen neuen Impuls zu geben. Gerade durch unseren Vorschlag können wir durch die Ergänzung und Kombination der Freiwilligen mit der Unfreiwilligen Feuerwehr den Brandschutz im Kreis auch langfristig aufrechterhalten."

\section{Kontra:}

Brandmeister Torben Seekamp

„Auf eine solche Idee können nur Menschen kommen, die keine Vorstellung davon haben, wie die Arbeit der freiwilligen Feuerwehr funktioniert. Für diejenigen, die tagtäglich für den Brandschutz aktiv sind, ist nicht vorstellbar, wie diese Leistung unfreiwillig erbracht werden soll. Zuverlässigkeit und Qualität der Arbeit wären insgesamt gefährdet - und das kann bestimmt niemand bei uns wollen!

Auch ist ein solcher Vorschlag für die vielen, hochmotivierten und engagierten Mitglieder der freiwilligen Feuerwehren ein Schlag ins Gesicht. Und ein solcher Vorschlag unterschätzt das Potenzial ehrenamtlichen Engagements bei uns im Kreis. Wir sind zuversichtlich, durch aktive Werbung unter den jüngeren Menschen und gerade auch durch die Angebote für die Incomings den Nachwuchssorgen, die zugegebenermaßen einige Wehren im Kreis haben, zu begegnen. Vorschläge wie der der unfreiwilligen Feuerwehr erschweren uns diese Arbeit völlig unnötigerweise."

Abb. 7.7 Spielmaterial für das Planspiel „Zukunftsdialog Multilokalität“(Quelle: TempALand 2020) 


\subsection{Strategien und Handlungsansätze zum Umgang mit Multilokalität}

Bisher ist wenig bekannt über den Alltag multilokal lebender Menschen in ländlichen Räumen. Informationen darüber sind für die Kommunen jedoch zunehmend von Bedeutung, um die Auswirkungen auf den Wohnungsmarkt, die Infrastrukturen, das Mobilitätsangebot oder das Engagement im Blick zu behalten und die Wünsche von Menschen mit mehreren Wohnsitzen besser berücksichtigen zu können. Für das Phänomen Multilokalität fehlt politischen und planerischen Akteur*innen zumeist noch das Bewusstsein, sodass sie für dieses Thema sensibilisiert werden sollten. Zudem ist es wichtig, Handlungsansätze und Strategien im Umgang mit Multilokalität zu entwickeln, denn es scheint noch unklar, wie verschiedene Akteur*innen auf das Phänomen Multilokalität reagieren können.

Im Rahmen der Untersuchungen des Projektes TempALand im Landkreis Diepholz wurden in transdisziplinären Entwicklungs- und Gestaltungsprozessen Maßnahmen und Projekte zusammen mit den lokalen Partner*innen entwickelt (siehe Abb. 7.8).

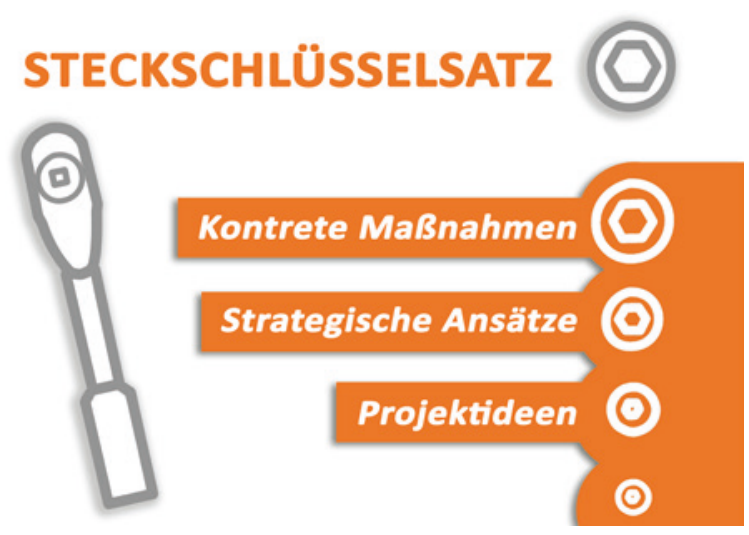

Abb. 7.8 Steckschlüsselsatz: Finden Sie passende Ideen zum Umgang mit Multilokalität (Quelle: TempALand 2020) 
Zwei zentrale Strategien für den Umgang mit Multilokalität wurden identifiziert:

- Attraktive Lebensbedingungen für Multilokale schaffen: Diese Strategie ist einerseits für die multilokal lebenden Personen interessant, kann aber andererseits auch für die lokale Bevölkerung attraktiv sein, weil dadurch Leistungen und Angebote ausgeweitet werden können. Im Zuge des steigenden Fachkräftemangels gewinnt sie auch für Unternehmen immer mehr an Bedeutung. Die Strategie stützt multilokale Lebensformen und will die Multilokalen sowohl Incomings wie Outgoings - in der jeweiligen Gemeinde halten.

- Multilokale sesshaft machen: Die Strategie zielt darauf, Multilokale - und ggf. ihre Familien oder Partner*innen - dauerhaft sesshaft zu machen (Incomings) bzw. ein Abwandern von Haushalten mit Multilokalen zu verhindern (Outgoings). Das kann neben den positiven kommunalfiskalischen Effekten für die Kommunen auch Chancen in Bezug auf das bürgerschaftliche Engagement der Multilokalen und den Kontakt zu Nachbarschaften bedeuten (vgl. Beitrag Greinke/Albrecht/Othengrafen/Gutsche/Lehmann in diesem Band (Kap. 5)). Die Strategie begreift Multilokalität als eine jeweils temporäre Lebensform und zielt darauf, sie zu beenden. Sie wird i. d. R. vor allem den Zielsetzungen von Kommunen, Unternehmen, Verbänden und Vereinen entsprechen. Bei entsprechender Zielerreichung trägt die Strategie zu einer Verringerung multilokal Lebender in der jeweiligen Gemeinde oder Stadt bei. Grundsätzlich trägt dies auch zur Steigerung der Attraktivität der Kommunen bei.

Beide Strategien wirken auf den ersten Blick als Alternativen, die sich gegenseitig ausschließen. Im Rahmen vom Projekt TempALand wurde jedoch deutlich, dass die beiden Strategien miteinander verknüpft werden können. Im ersten Schritt geht es v. a. Kommunen darum, Multilokalen (Incomings) ein attraktives Lebensumfeld zu bieten, um sie im zweiten Schritt davon zu überzeugen, dauerhaft in die Gemeinde oder Stadt zu ziehen und dort sesshaft zu werden.

Im Projekt TempALand wurden, zusammen mit den lokalen Akteur*innen insgesamt 39 konkrete Maßnahmen und Projektideen entwickelt (siehe Abb. 7.9). Zusammengefasst als „Steckschlüsselsatz“ sind sie unter www.tempaland.de detailliert dargestellt. Neben einer Sortierung nach Handlungsfeldern

- (1) Wohnen,

- (2) technische und soziale Infrastrukturen,

- (3) Unternehmen, und

- (4) bürgerschaftliches Engagement 

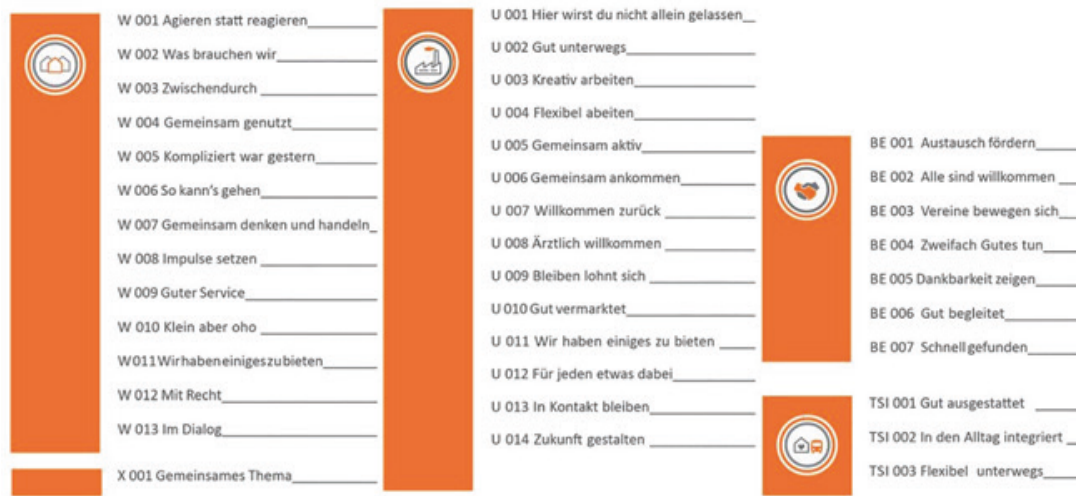

Abb. 7.9 Gesamtübersicht der 39 Maßnahmen und Projektideen (Quelle: TempALand 2020)

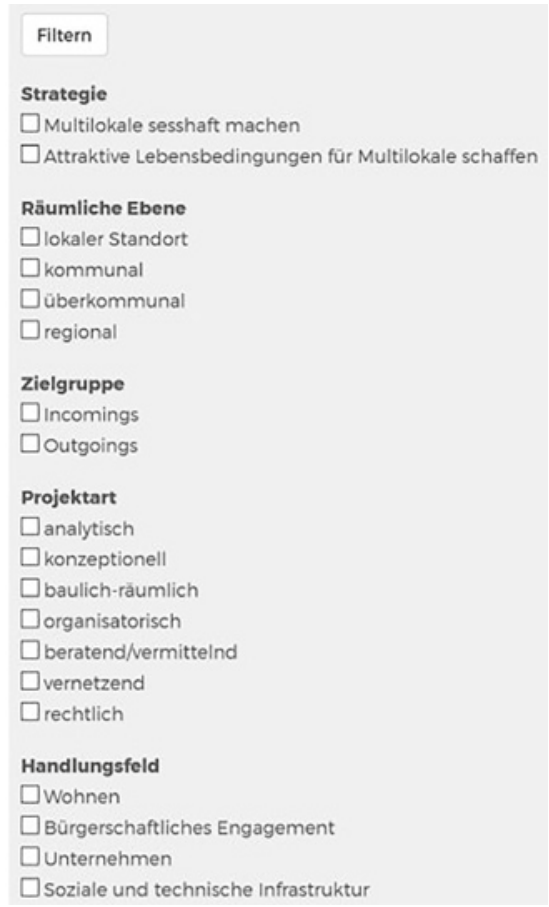

Abb. 7.10 Filtermaske des Steckschlüsselsatzes (Quelle: TempALand 2020) 
kann das digitale Tool auch die Maßnahmen und Projektideen differenziert nach der Zielgruppe (Incomings/Outgoings), der räumlichen Ebene (lokal, kommunal, überkommunal, regional) sowie der Projektart suchen und zusammenfassen (siehe Abb. 7.10). Im Folgenden werden die verschiedenen Projektarten - analytische, konzeptionelle, baulich-räumliche, organisatorisch-vernetzende, beratende und vermittelnde sowie rechtliche Projekte - im Kontext von Multilokalität kurz beschrieben und mit Beispielen erläutert.

\section{Analytische Projekte}

Wie in den vorangegangenen Kapiteln beschrieben, ist der Kenntnisstand zur Multilokalität oft eingeschränkt. Vor diesem Hintergrund gewinnen analytische Maßnahmen an Bedeutung, um das Phänomen Multilokalität „greifbar" zu machen und zu analysieren. Diese Bedeutung wurde im Rahmen des TempALand-Projektes u. a. bei den Planspielen betont. Analytische Projekte von TempALand können sich dabei zum einen ausschließlich mit Multilokalität beschäftigen, zum anderen Multilokalität bei anderen Themen mit einbinden.

\section{Ermittlung des Bedarfs an Zweitwohnungen}

Als Voraussetzung für eine Überprüfung, inwieweit das lokale und regionale Wohnungsangebot aus Sicht der Multilokalen „,bedarfsgerecht" ist, wird der bestehende Bedarf in Annäherung ermittelt. Durch die erhobenen Daten zum Bedarf an Wohnraum können Kommunen (und Unternehmen) die Wohnungsmarktentwicklungen entsprechend beobachten und ggf. steuernd eingreifen. Dadurch kann angespannten Wohnungsmärkten vorgebeugt werden. Die Bedarfe der Multilokalen sind dabei so breit gefächert wie die Motivationen und Ursachen der Multilokalität. Im ersten Ansatz wird dem Wohnbedarf von Menschen nachgegangen, die aus Arbeitsgründen temporär im Landkreis sind (Incomings). Dies kann eine Diversifizierung des Wohnungsmarktes zur Folge haben. Wird das Ziel der Innen- vor Außenverdichtung dabei umgesetzt, können außerdem Ortskerne bewahrt und der Gebäudebestand in andere Nutzungen überführt werden.

Die Bedarfsermittlung kann eine Grundlage kommunaler Wohnbaukonzeptionen sein. Zielgruppe der Maßnahme sind die Incomings; die Maßnahme zielt darauf, attraktive Lebensbedingungen für Multilokale zu schaffen und ist von daher sowohl für Kommunen wie für Unternehmen 
interessant. Die Ermittlung des Bedarfs an Zweit(miet)wohnungen kann dabei auf kommunaler, auf überkommunaler oder regionaler Ebene stattfinden. Mögliche Wege zur Umsetzung dieser Maßnahme sind u. a. telefonische Befragungen der ansässigen Unternehmen zur Anzahl Multilokaler im Betrieb und deren Bedarf an Zweitwohnungen. Eine Kooperation von Kommunen und Unternehmen bietet sich daher an. Die Ergebnisse dieses analytischen Schrittes können als Grundlage konzeptioneller und strategischer Überlegungen genutzt werden.

\section{Konzeptionelle Projekte}

Konzeptionelle Projekte im Kontext von Multilokalität werden i.d. R. ein breiteres Themenspektrum als Multilokalität umfassen, z. B. in den Themenfeldern Wohnen oder Mobilität. Ihnen gehen i.d.R. analytische Arbeitsschritte voraus (oder sind Bestandteil von ihnen) bspw. zur Ermittlung des Bedarfs oder von Ansprüchen. In den meisten Fällen wird die öffentliche Hand Trägerin der Projekte sein. Eine Einbindung weiterer Akteursgruppen (je nach Thema: Wohnungswirtschaft, Verkehrsbetriebe, Unternehmen) ist dabei sinnvoll, vor allem, wenn in einer Umsetzung der erarbeiteten Konzeption die Mitwirkung dieser Akteursgruppen erforderlich ist.

\section{Regionale Wohnbaukonzeption}

Eine Wohnbaukonzeption greift aktuelle Bevölkerungsprognosen auf, verfolgt das Ziel, den zukünftigen Wohnraumbedarf konkret und greifbar zu machen und schreibt Leitziele für die zukünftige Entwicklung des Wohnungsmarktes der Kommune fest. Zudem entwickelt

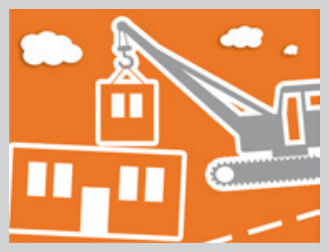
sie eine Umsetzungsstrategie. Themen sind dabei sowohl der Bestand als auch der Neubau. Durch den Neubau kann der Wohnungsmarkt vielseitiger gestaltet werden, während der Bestand bei geschickter Anwendung nach- oder umgenutzt werden kann. Die Zielgruppe der multilokalen Incomings wird u. a. beim Thema Bedarf an kleinem Wohnraum berücksichtigt. Durch eine Wohnbaukonzeption wird die zielgerichtete Wohnungsmarktentwicklung ermöglicht, Maßnahmen und Projekte können abgestimmt vorbereitet werden. Eine Wohnbaukonzeption kann sowohl dafür genutzt werden, Multilokalen, die als 
Incomings ihre multilokale Lebensweise beibehalten werden, attraktiven Wohnraum zu bieten als auch dafür, Multilokalen, die in der Kommune sesshaft werden wollen, attraktive Wohnangebote zu machen. Von daher kann sie die beiden Strategien (Multilokale sesshaft machen, attraktive Lebensbedingungen für Multilokale schaffen) verfolgen. Eine Wohnbaukonzeption kann auf kommunaler, überkommunaler und regionaler Ebene umgesetzt werden. Die regionale Ebene ist dabei im Kontext von Multilokalität wichtig, da Multilokalität sich nicht an administrativen Grenzen orientiert. Zumindest sollte geprüft werden, inwieweit die gemeinsame Entwicklung für mehrere Nachbargemeinden sinnvoll ist.

\section{Baulich-räumliche Projekte}

Baulich-räumliche Projekte sind Projekte, die räumlich verortet und konkret baulich umgesetzt werden. Dabei liegt der Fokus im Kontext von Multilokalität im Themenbereich Wohnen zumeist auf den Incomings, um für diese Zielgruppe geeignete Angebote zu schaffen. denn durch ein bedarfsgerechtes und attraktives Wohnangebot kann die Attraktivität der Region z. B. für multilokale Fachkräfte steigen. Durch die (neuen) baulich-räumlichen Angebote können der Wohnungsmarkt und touristische Angebote (z. B. Hotels) sinnvoll ergänzt werden, ohne Konkurrenzen zu bilden.

\section{Entwicklung eines zentralen und multifunktio- nalen 'Servicewohnhauses'}

Ein zentrales und multifunktionales „Servicewohnhaus" ist eine Art Hotel, in dem individuelle Wohneinheiten in unterschiedlichen Größen mit flexibler Mietdauer angemietet werden können. Die Wohnein-

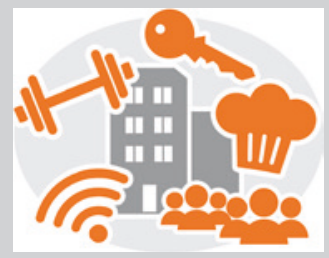
heiten sind teilweise möbliert und werden durch Gemeinschaftsräume (z. B. Gemeinschaftsküche, Waschküche, Aufenthaltsraum, Sportraum etc.) ergänzt. Das Servicewohnhaus stellt weitere Dienstleistungen (z. B. Hausverwaltung, Putzservice etc.) oder Infrastrukturen (z. B. WLAN) zur Verfügung. Die Idee lehnt an das Konzept des „Commonhousing“ an und ist für verschiedene Zielgruppen (z. B. 
Studierende, multilokal lebende Arbeitskräfte, aber auch ggf. Tourist*innen etc.) attraktiv. Das Servicewohnhaus kann als „Netzwerkpunkt“ fungieren, an dem sich die Zielgruppen treffen und austauschen. Die vielfältigen Wohnmöglichkeiten eines Servicewohnhauses unterstützen eine Diversifizierung des Wohnungsmarktes.

Mit einem Servicewohnhaus können attraktive Lebensbedingungen für Multilokale, insbesondere Incomings, geschaffen werden. Projektträger*innen können entweder Kommunen oder auch private Investor*innen sein. Als mögliche Projektpartner*innen kommen Akteur*innen aus der Wohnungswirtschaft, Unternehmen, lokalen Bevölkerung, Multilokale und Investor*innen in Frage.

Für die Umsetzung sollten zunächst ein geeignetes Grundstück identifiziert und geeignete Bestandsgebäude geprüft werden. Multilokale sollten bei Planung und Umsetzung beteiligt werden, um ihre Wünsche und Ideen aufgreifen zu können.

\section{Beratende und vermittelnde Projekte}

Projekte mit Beratungs- und Vermittlungsfunktion haben das Ziel, Multilokale und ihre Haushalte in verschiedenen Fragen und Lebensbereichen zu unterstützen. Dabei kann es zum einen darum gehen, den Multilokalen den Alltag zu erleichtern, zum anderen auch darum, Multilokale „sesshaft“ zu machen. Die Projekte arbeiten daran, Informationen und Angebote besser an mögliche Zielgruppen zu vermitteln und dadurch zum einen die Multilokalen zu unterstützen und zum anderen vorhandene Angebote besser auszunutzen. Dabei kann es um sehr verschiedene Bereiche gehen - Arbeitsplätze, Wohnraum, soziale Infrastruktur u. a.m.

\section{Vermittlung von Arbeitsplätzen für Partner- innen und Partner}

Unternehmen vermitteln in Kooperation mit Kommunen Arbeitsplätze für die Partnerin oder den Partner der Multilokalen. Dadurch erhöht sich die Chance, den gesamten Haushalt sesshaft zu machen. Zielgruppe sind multi-

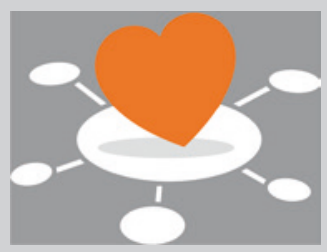
lokale Incomings, die sesshaft werden und ihre Partnerin oder ihren Partner bzw. ihre Familie „nachholen“ wollen. Die 
Projektidee kann dadurch auch einen Beitrag zur Gewinnung bzw. Bindung von Fachkräften leisten. Die Vermittlung von Arbeitsplätzen ist am lokalen Standort der Unternehmen sowie kommunal und überkommunal möglich. Multilokale Incomings können durch die Unternehmen angesprochen werden. Die Unterstützung bei der Arbeitssuche von Partnerinnen und Partnern kann durch Unternehmen in Kooperation mit Kommunen „beworben“ werden. Zudem ist die Einrichtung einer Anlaufstelle für arbeitssuchende Partnerinnen und Partner bei der Kommune sinnvoll. Darüber hinaus sollten Kommunen und Unternehmen sich untereinander vernetzen, um Informationen über „passende“, freie Stellen zügig verfügbar zu haben.

\section{Organisatorische und vernetzende Projekte}

Organisatorische und vernetzende Projekte wollen durch Austausch, Koordination, Kommunikation und Kooperation verschiedene Akteur*innen zusammen bringen. Ziel ist es u.a. ein gemeinsames Verständnis von Zielen und Aktivitäten im Hinblick auf verschiedene Themen, z.B. Wohnraumversorgung oder bürgerschaftliches Engagement oder auch Infrastrukturen zu schaffen. Gemeinsame Absprachen und Zielvorgaben können gute Rahmenbedingungen für eine zügige und zielgerichtete Umsetzung von Projekten ermöglichen. Multilokalität kann dabei entweder Ursache und Ausgangspunkt für die Vernetzungsprojekte sein oder ein Thema in größerem Zusammenhang. Durch organisatorische Projekte können Multilokale, z.B. Studierende, angezogen bzw. „,vom Bleiben überzeugt“ werden und die Region kann an Attraktivität und Zuzug gewinnen. Unternehmen vor Ort können ggf. leichter Fachkräfte gewinnen.

\section{Regionales Bündnis für Wohnen}

Die Bewältigung der aktuell vielfältigen und dringenden Aufgaben im Bereich des Wohnungsmarktes legt es nahe, die Zusammenarbeit der verschiedenen Akteur*innen (insbesondere aus Kommunen und Wohnungswirtschaft) $\mathrm{zu}$ intensivieren sowie ihre Hand-

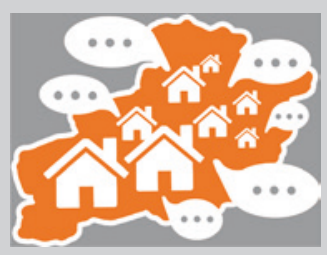
lungsmöglichkeiten und Spielräume $\mathrm{zu}$ koordinieren. Mit Vertreter*innen des Landkreises, der Kommunen und der Wohnungswirtschaft wird ein „Bündnis für Wohnen“ ins Leben 
gerufen. $\mathrm{Zu}$ den Aufgaben des Bündnisses gehören die Intensivierung der Zusammenarbeit zwischen Kommunen und Wohnungswirtschaft ebenso wie die Intensivierung der überkommunalen Zusammenarbeit im Bereich des Wohnungsmarktes. Auch das Vorantreiben der Umsetzung konkreter Projekte kann zum Tätigkeitsspektrum gehören. Im Kern geht es um die Realisierung eines differenzierten, bedarfsgerechten Wohnangebotes in der Region, um ein passendes Angebot u. a. für multilokale Incomings zu schaffen. Dies hat auch eine Diversifizierung des Wohnungsmarktes zur Folge. Vielerorts fehlen gerade kleinere Wohnungen, die nicht nur für Incomings, sondern auch z. B. für ältere, alleinstehende Menschen von Interesse sind.

Die Projektidee zielt darauf ab, auf überkommunaler und regionaler Ebene Incomings und Outgoings sesshaft zu machen sowie attraktive Lebensbedingungen für sie zu schaffen. Für die Umsetzung sollte zunächst der Bedarf eines regionalen Bündnisses für Wohnen ermittelt werden. Danach können Landkreise durch die Einladung möglicher Interessierten zu einem ersten Informations- und Austauschgespräch erste Impulse setzen. Gemeinsam sollten Zielsetzung und Aufgabenstellung sowie die Rollenverteilung für das regionale Bündnis für Wohnen geklärt werden, bevor eine Verständigung auf ein gemeinsames Leitbild stattfindet und ein konkretes Arbeitsprogramm gemeinsam entwickelt wird.

\section{Rechtliche Projekte}

Rechtliche Projekte im Kontext mit Multilokalität nutzen (planungs-)rechtliche Vorgaben und Bedingungen dafür, dem Bedarf von Multilokalen besser Rechnung zu tragen. Ggf. ist im Vorfeld eine Sensibilisierung der jeweils Zuständigen (im Regelfall die Kommunen) möglich, um sie auf die rechtlichen Möglichkeiten und Chancen in diesem Feld aufmerksam zu machen. Im Fokus steht dabei zumeist das Themenfeld Wohnen. Es ist aber auch möglich, dass Rahmenbedingungen in anderen Kontexten, z.B. in Vereinen angepasst werden. Durch die Veränderungen können verschiedene Nutzer*innengruppen, wie Multilokale (Incomings), angesprochen werden. Im Vergleich zu den bisher genannten Projektarten erreichen rechtliche Projekte die größte Verbindlichkeit. Ihre Anwendung wird daher in vielen Fällen die Folge z.B. analytischer und konzeptioneller Projekte bzw. Arbeitsschritte sein. 
Anpassung planungsrechtlicher Voraussetzungen und Vergabekriterien für Bauvorhaben im kommunalen Eigentum

Bei der Bebauung von Flächen in kommunalem Eigentum haben die Kommunen die größten Einflussmöglichkeiten. Sofern sie nicht selber bauen, können sie die Bebauung über Vergabe$(0)=7$
$5 \mathrm{~g}=\mathrm{S}=\mathrm{O}$ kriterien und die Art der Neubebauung mitbestimmen. Dadurch können sie an geeigneten Standorten die Errichtung kleineren, flexibleren und anpassungsfähigen Wohnraums fördern, um einen Beitrag zur Diversifizierung von homogenen Wohnungsmärkten zu leisten. Darüber hinaus könnten für diese Kriterien neue planungsrechtliche Voraussetzungen geschaffen werden. Kommunen können dies bspw. durch entsprechende Festsetzungen in der Bauleitplanung steuern. Hierdurch kann auf dem Wohnungsmarkt besser auf die Bedarfe multilokal lebender Menschen reagiert werden. Eine Kombination mit einem Wettbewerb ist möglich.

Um der Zielgruppe der Incomings gerecht zu werden und für Multilokale attraktive Lebensbedingungen $\mathrm{zu}$ schaffen, ist auf kommunaler Ebene zunächst zu prüfen, ob Kommunen geeignete Grundstücke besitzen und ob die Grundstücke behalten, veräußert oder in Erbpacht vergeben werden sollen. Darüber hinaus können allgemeine oder standortspezifische Vergabekriterien sowie planungsrechtliche Voraussetzungen geprüft werden. Zudem sollten diese bei der Neuaufstellung von Bebauungsplänen berücksichtigt werden.

\subsection{Zukünftige Entwicklungen und temporäre An- und Abwesenheiten}

Multilokalität als Massenphänomen aller Altersgruppen und sozialen Schichten (Weichhart 2015: 378; ARL 2016: 7; Oberösterreichische Akademie 2019: 4) steht bisher und wird zukünftig in engem Zusammenhang mit gesamtgesellschaftlichen sozialen, wirtschaftlichen und technischen Entwicklungen stehen. Dies gilt sowohl für urbane wie auch für ländliche Räume. Insbesondere in ländlichen Räumen kann Multilokalität mit den vielfältigen Erscheinungsformen unter- 
schiedliche gesellschaftliche und (sozial-)räumliche Auswirkungen bedeuten und dabei Herausforderung als auch Chance für die Stadt- und Regionalplanung sein, u. a. wenn entsprechende Wohnungsangebote geschaffen werden (müssen) oder Incomings zumindest zeitweise als Fachkräfte gewonnen werden können (vgl. Beitrag Othengrafen/Greinke/Danielzyk in diesem Band (Kap. 6)).

Die Analysen im Landkreis Diepholz haben wichtige Erkenntnisse für den Umgang mit Multilokalität generiert. Ein zentrales Ergebnis ist, dass die Wahrnehmung des Phänomens bislang noch unzureichend ist. Aus diesem Grund ist die Sensibilisierung für Multilokalität und die damit zusammenhängenden Auswirkungen (zukünftig) wichtig. Die vorgeschlagenen Strategien und Projekte bilden erste Ansätze zum Umgang mit Multilokalität. Oft decken sich die Maßnahmen und Strategien zudem mit bereits bestehenden Herausforderungen, denen dadurch begegnet werden kann. Dazu zählen zum Beispiel Auswirkungen der Pendler*innenmobilität, des demografischen Wandels oder auch Nutzungszyklen von Wohnungen sowie viele weitere (vgl. Beitrag Greinke/Albrecht/ Othengrafen/Gutsche/Lehmann in diesem Band (Kap. 5)). Multilokalität kann folglich in vielen Räumen ein „verstärkendes Element“ sein und die bereits bestehenden Rahmenbedingungen betonen, zum Beispiel in eher peripheren ländlichen Räumen mit negativer Bevölkerungsentwicklung oder weiteren Herausforderungen, sodass darauf reagiert werden muss (vgl. Beitrag Othengrafen/ Greinke/Danielzyk in diesem Band (Kap. 6)). Zukünftig sollte deshalb Multilokalität als Thema in das Verwaltungshandeln der Kommunen sichtbar integriert werden, um zukünftigen Herausforderungen entgegen zu treten.

Es ist absehbar, dass dass die voranschreitende Digitalisierung sowie die Entwicklung und Pluralisierung der Lebensstile Multilokalität weiter beeinflussen werden: Neben neuen Informations- und Kommunikationsdienstleistungen werden zukünftig auch neue, digitale Mobilitätsdienstleistungen räumliche Auswirkungen bedingen. Rückblickend können Menschen aktuell bereits deutlich weitere Strecken in kürzerer Zeit zurücklegen. Bei weiterem und schnellerem Ausbau dieser Infrastruktur sind somit ggf. noch deutlich weitere Entfernungen in kürzester Zeit zurückzulegen, wodurch sich auch die Wohn- und Arbeitsorte der Menschen deutlich verändern können. Denkbar ist, dass Multilokale dann ihre Pendelhäufigkeit vergrößern und somit sogar zu täglichen Pendler*innen werden können (vgl. Greinke 2020), weil eine weitere Unterkunft aufgrund der ausgebauten Anbindung nicht mehr nötig ist.

Darüber hinaus lässt sich vermuten, dass mit voranschreitendem Ausbau des Breitbandausbaus, v. a. in ländlichen Räumen, „neue“ Arbeitsformen möglich 
sind. Die in urbanen Räumen bereits etablierten Co-Working-Spaces können durch die Digitalisierung auch in ländlichen Räumen eine Chance sein. Leben in einer Kommune zum Beispiel viele Outgoings, die potenziell zumindest einen Teil ihrer Arbeit im Co-Working-Space ausüben könnten, würden die Outgoings die Kommunen weniger oft verlassen müssen. Das kann dazu führen, dass die multilokalen Outgoings an ihrem weiteren (Arbeits-)Ort keine Unterkunft mehr benötigen, weil sie nur für sehr kurze Zeiträume, zum Beispiel in Form von Dienstreisen, an den Arbeitsort reisen müssen. Dadurch halten sich die Outgoings länger in ihrer Ausgangskommune auf, nutzen die dortigen sozialen und technischen Infrastrukturen und stehen potenziell auch für lokale Gemeinschaften und Engagementtätigkeiten zur Verfügung.

Des Weiteren können Automatisierungsprozesse für veränderte multilokale Lebensweisen sorgen. Technologien können eine Chance für ländliche Räume sein (Jacoby/Wappelhorst 2016: 95). Wenn beispielsweise hochautomatisierte Fahrzeuge etabliert würden, könnten Fahrzeuge neben dem reinen Transportmittel, wie es Pkw derzeit noch sind, auch andere Funktionen übernehmen. Denkbar ist, dass auf den Reisen zwischen den Orten einer beruflichen Tätigkeit, z. B. im mobilen Büro, nachgegangen werden kann. Dadurch würden Multilokale weniger Fahrtzeit „vergeuden“. Vorstellbar ist, dass das zu einer Zunahme mehrörtiger Lebensweisen führt, weil die Menschen während der Fahrtzeit andere Tätigkeiten ausüben und weitere Entfernungen zurücklegen können.

Außerdem können verändertes Mobilitätsverhalten, -anforderungen und -möglichkeiten, wie der Trend zum Sharing, mehrörtige Lebensweisen beeinflussen. Vorstellbar ist, dass Multilokale in ländlichen Räumen nicht mehr einen privaten PKW für die An- und Abreisen nutzen, sondern neben reinen Fahrgemeinschaften auf Sharingangebote ausweichen und somit keinen eigenen Pkw (mehr) benötigen. Das ist nicht nur aus der Perspektive einer nachhaltigen Transformation sinnvoll, sondern es kann auch soziale Kontakte fördern. Durch Sharingangebote kommen Multilokale mit anderen Nutzenden in Kontakt und können ihre Netzwerke ausbauen.

Die in diesem Kapitel beispielhaft aufgezeigten Handlungsempfehlungen können eine gute Grundlage für zukünftiges politisches und planerisches Handeln sein. Aufgrund der empirischen Herleitung und der Entwicklung mit lokalen Akteur*innen sind sie insbesondere für den Landkreis Diepholz reliabel und valide. Sie lassen sich aber nach einer Überprüfung auch auf andere ländliche Räume übertragen.

Zukünftig sollten multilokale Lebensweisen in Forschung und kommunaler Praxis weiter in den Fokus rücken. Dafür ist eine tief greifende Auseinandersetzung mit der Thematik multilokaler Lebenspraktiken nötig. Wissenschaftliche 
und anwendungsorientierte Analysen sollten dabei in inter- und transdisziplinären Teams angelegt werden. Die Facetten und Ausprägungen mehrörtiger Lebensformen sind derart divers und fluide, dass es erforderlich ist, sie mit qualitativen und quantitativen Instrumenten und Methoden der Datenerhebung zu untersuchen. Unter anderem fehlen Aussagen zur Grundgesamtheit multilokaler Lebensweisen, aber auch vergleichende (internationale) Studien in unterschiedlichen räumlichen Maßstäben und Kontexten (vgl. Beitrag Albrecht/Dittrich-Wesbuer in diesem Band (Kap. 3)). Sowohl die unterschiedlichen Motive und Anlässe als auch die diversen Wechselwirkungen zwischen multilokalen Lebensweisen, gesellschaftlichen und räumlichen Auswirkungen sowie planerischen bzw. politischen Steuerungsmöglichkeiten sollten vertiefend fokussiert werden, um daraus politische und planerische Handlungsempfehlungen für den Umgang mit multilokalen Lebensweisen abzuleiten, damit eine nachhaltige Raum-, Regional- und Stadtentwicklung in urbanen und ländlichen Räumen umgesetzt werden kann. Multilokalität als Phänomen und die damit verbundenen Folgewirkungen und Potenziale gilt es dabei stets mitzudenken (vgl. Beiträge Greinke/Lange/Born (Kap. 2), Greinke/Lange (Kap. 4) und Greinke/Albrecht/Othengrafen/Gutsche/Lehmann (Kap. 5) in diesem Band).

\section{Literatur}

ARL - Akademie für Raumforschung und Landesplanung (Hg.) (2016): Multilokale Lebensführung und räumliche Entwicklungen. Positionspapier aus der ARL 104. Hannover.

Greinke, L. (2020): Berufsbedingte Multilokalität in ländlichen Räumen Niedersachsens. Gesellschaftliche und räumliche Auswirkungen als planerische Herausforderung am Beispiel des Landkreises Diepholz. Ländliche Räume: Beiträge zur lokalen und regionalen Entwicklung Bd. 7, LIT-Verlag Berlin.

Jacoby, C./Wappelhorst, S. (Hg.) (2016): Potenziale neuer Mobilitätsformen und -technologien für eine nachhaltige Raumentwicklung. Arbeitsberichte der ARL 18. Hannover.

Landkreis Diepholz (LK DH) (2016): Wohnraumversorgungskonzept Landkreis Diepholz. Diepholz.

Oberösterreichische Zukunftsakademie (Hg.) (2019): Leben an mehreren Orten. Multilokalität als Chance für Stadt und Land. Linz.

Weichhart, P./Rumpolt, P. A. (Hg.) (2015): Mobil und doppelt sesshaft. Studien zur residenziellen Multilokalität. Wien: Abhandlungen zur Geographie und Regionalforschung Bd. 18 . 
Open Access Dieses Kapitel wird unter der Creative Commons Namensnennung 4.0 International Lizenz (http://creativecommons.org/licenses/by/4.0/deed.de) veröffentlicht, welche die Nutzung, Vervielfältigung, Bearbeitung, Verbreitung und Wiedergabe in jeglichem Medium und Format erlaubt, sofern Sie den/die ursprünglichen Autor(en) und die Quelle ordnungsgemäß nennen, einen Link zur Creative Commons Lizenz beifügen und angeben, ob Änderungen vorgenommen wurden.

Die in diesem Kapitel enthaltenen Bilder und sonstiges Drittmaterial unterliegen ebenfalls der genannten Creative Commons Lizenz, sofern sich aus der Abbildungslegende nichts anderes ergibt. Sofern das betreffende Material nicht unter der genannten Creative Commons Lizenz steht und die betreffende Handlung nicht nach gesetzlichen Vorschriften erlaubt ist, ist für die oben aufgeführten Weiterverwendungen des Materials die Einwilligung des jeweiligen Rechteinhabers einzuholen.

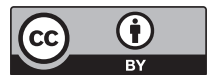

